\title{
REVERSE LEXICOGRAPHIC SQUAREFREE INITIAL IDEALS AND GORENSTEIN FANO POLYTOPES
}

\author{
HIDEFUMI OHSUGI AND TAKAYUKI HIBI
}

\begin{abstract}
Via the theory of reverse lexicographic squarefree initial ideals of toric ideals, we give a new class of Gorenstein Fano polytopes (reflexive polytopes) arising from a pair of stable set polytopes of perfect graphs.
\end{abstract}

\section{INTRODUCTION}

Recall that an integral convex polytope is a convex polytope all of whose vertices have integer coordinates. An integral convex polytope $\mathcal{P} \subset \mathbb{R}^{d}$ of dimension $d$ is called a Fano polytope if the origin of $\mathbb{R}^{d}$ is a unique integer point belonging to the interior of $\mathcal{P}$. We say that a Fano polytope $\mathcal{P} \subset \mathbb{R}^{d}$ is Gorenstein if the dual polytope $\mathcal{P}^{\vee}$ of $\mathcal{P}$ is again integral. (A Gorenstein Fano polytope is often called a reflexive polytope in the literature.) Gorenstein Fano polytopes are related with mirror symmetry and studied in a lot of areas of mathematics. See, e.g., [5, §8.3] and [15. It is known that there are only finitely many Gorenstein Fano polytopes up to unimodular equivalence if the dimension is fixed. Classification results are known for low dimensional cases [16, 17]. On the other hand, one of the most important problem is to construct new classes of Gorenstein Fano polytopes. In the case of Gorenstein Fano simplices, there are nice results on classifications and constructions. See, e.g., [4, 14, 18] and their references. In order to find classes of Gorenstein Fano polytopes of high dimension which are not necessarily simplices, integral convex polytopes arising from some combinatorial objects are studied in several papers. For example, the following classes are known:

- Gorenstein Fano polytopes arising from the order polytopes of graded posets (Hibi [10, revisited by Hegedüs-Kasprzyk [7, Lemma 5.10]);

- Gorenstein Fano polytopes arising from the Birkhoff polytopes (appearing in many papers. See, e.g., Stanley's book [24, I.13] and Athanasiadis [1]);

- Gorenstein Fano polytopes arising from directed graphs satisfying some conditions (Higashitani [13]);

- Centrally symmetric configurations (Ohsugi-Hibi [23]);

- The centrally symmetric polytope $\mathcal{O}(P)^{ \pm}$of the order polytope $\mathcal{O}(P)$ of a finite poset $P$ (Hibi-Matsuda-Ohsugi-Shibata [12]).

2010 Mathematics Subject Classification. Primary 13P10; Secondary 52B20.

Key words and phrases. Gröbner basis, Gorenstein Fano polytope, unimodular triangulation. 
In the present paper, via the theory of Gröbner bases, we give a new class of Gorenstein Fano polytopes which is not necessarily a simplex. For any pair of perfect graphs $G_{1}$ and $G_{2}$ (here, $G_{1}=G_{2}$ is possible) on $d$ vertices, we show that the convex hull of $\mathcal{Q}_{G_{1}} \cup-\mathcal{Q}_{G_{2}}$, where $\mathcal{Q}_{G_{i}}$ is the stable set polytope of $G_{i}$, is a Gorenstein Fano polytope of dimension $d$. Note that there are a lot of pairs of perfect simple graphs on $d$ vertices 1 (Figure 1 ). Any Gorenstein Fano polytope $\mathcal{P}$ in our class is

\begin{tabular}{|c|c|c|c|c|c|c|c|}
\hline number of vertices & 2 & 3 & 4 & 5 & 6 & 7 & 8 \\
\hline perfect graphs & 2 & 4 & 11 & 33 & 148 & 906 & 8887 \\
\hline pairs of perfect graphs & 3 & 10 & 66 & 561 & 11,026 & 410,871 & $39,493,828$ \\
\hline
\end{tabular}

FiguRE 1. Number of perfect graphs / pairs of perfect graphs

terminal, i.e., each integer point belonging to the boundary of $\mathcal{P}$ is a vertex of $\mathcal{P}$. In particular, if both of two graphs are the complete (resp. empty) graphs on $d$ vertices, then the Gorenstein Fano polytope has $2 d$ (resp. $2^{d+1}-2$ ) vertices. Thus, our class has enough size and variety comparing with the existing classes above.

Let $\mathbb{Z}_{\geq 0}$ denote the set of nonnegative integers. Let $A=\left[\mathbf{a}_{1}, \ldots, \mathbf{a}_{n}\right] \in \mathbb{Z}_{\geq 0}^{d \times n}$ and $B=\left[\mathbf{b}_{1}, \ldots, \mathbf{b}_{m}\right] \in \mathbb{Z}_{\geq 0}^{d \times m}$, where each $\mathbf{a}_{i}$ and each $\mathbf{b}_{j}$ is a nonzero column vector belonging to $\mathbb{Z}_{\geq 0}^{d}$. In Section 1 , after reviewing basic materials and notation on toric ideals, we introduce the concept that $A$ and $B$ are of harmony. Roughly speaking, Theorem 1.1 says that if $A$ and $B$ are of harmony and if the toric ideal of each of $A$ and $B$ possesses a reverse lexicographic squarefree initial ideal which enjoys certain properties, then the toric ideal of $[\mathbf{0},-B, A] \in \mathbb{Z}^{d \times(n+m+1)}$ possesses a squarefree initial ideal with respect to a reverse lexicographic order whose smallest variable corresponds to the column $\mathbf{0} \in \mathbb{Z}^{d}$. Working with the same situation as in Theorem 1.1, Corollary 1.3 guarantees that if the integral convex polytope $\mathcal{P} \subset \mathbb{R}^{d}$ which is the convex hull of $\left\{-\mathbf{b}_{1}, \ldots,-\mathbf{b}_{m}, \mathbf{a}_{1}, \ldots, \mathbf{a}_{n}\right\}$ is a Fano polytope with $\mathcal{P} \cap \mathbb{Z}^{d}=\left\{\mathbf{0},-\mathbf{b}_{1}, \ldots,-\mathbf{b}_{m}, \mathbf{a}_{1}, \ldots, \mathbf{a}_{n}\right\}$ and if there is a $d \times d$ minor $A^{\prime}$ of $[-B, A]$ with $\operatorname{det}\left(A^{\prime}\right)= \pm 1$, then $\mathcal{P}$ is Gorenstein.

The topic of Section 2 is the incidence matrix $A_{\Delta}$ of a simplicial complex $\Delta$ on $[d]=\{1, \ldots, d\}$. It follows that if $\Delta$ and $\Delta^{\prime}$ are simplicial complexes on $[d]$, then $A_{\Delta}$ and $A_{\Delta^{\prime}}$ are of harmony. Following Theorem 1.1 it is reasonable to study the problem when the toric ideal of $A_{\Delta}$ satisfies the required condition on initial ideals of Theorem 1.1. Somewhat surprisingly, Theorem 2.6 says that $A_{\Delta}$ satisfies the required condition on initial ideals of Theorem 1.1 if and only if $\Delta$ coincides with the set $S(G)$ of stable sets of a perfect graph $G$ on $[d]$. A related topic on Gorenstein Fano polytopes arising from simplicial complexes will be studied (Theorem 2.8).

\footnotetext{
${ }^{1}$ See A052431 in "The On-Line Encyclopedia of Integer Sequences," at http://oeis.org
} 


\section{REVERSE LEXICOGRAPHIC SQUAREFREE INITIAL IDEALS}

Let $K$ be a field and $K\left[\mathbf{t}, \mathbf{t}^{-1}, s\right]=K\left[t_{1}, t_{1}^{-1}, \ldots, t_{d}, t_{d}^{-1}, s\right]$ the Laurent polynomial ring in $d+1$ variables over $K$. Given an integer $d \times n$ matrix $A=\left[\mathbf{a}_{1}, \ldots, \mathbf{a}_{n}\right]$, where $\mathbf{a}_{j}=\left[a_{1 j}, \ldots, a_{d j}\right]^{\top}$, the transpose of $\left[a_{1 j}, \ldots, a_{d j}\right]$, is the $j$ th column of $A$, the toric ring of $A$ is the subalgebra $K[A]$ of $K\left[\mathbf{t}, \mathbf{t}^{-1}, s\right]$ which is generated by the Laurent polynomials $\mathbf{t}^{\mathbf{a}_{1}} s=t_{1}^{a_{11}} \cdots t_{d}^{a_{d 1}} s, \ldots, \mathbf{t}^{\mathbf{a}_{n}} s=t_{1}^{a_{1 n}} \cdots t_{d}^{a_{d n}} s$. Let $K[\mathbf{x}]=K\left[x_{1}, \ldots, x_{n}\right]$ denote the polynomial ring in $n$ variables over $K$ and define the surjective ring homomorphism $\pi: K[\mathbf{x}] \rightarrow K[A]$ by setting $\pi\left(x_{j}\right)=\mathbf{t}^{\mathbf{a}_{j}} s$ for $j=1, \ldots, n$. The toric ideal of $A$ is the kernel $I_{A}$ of $\pi$. Every toric ideal is generated by binomials. (Recall that a polynomial $f \in K[\mathbf{x}]$ is a binomial if $f=u-v$, where $u=\prod_{i=1}^{n} x_{i}^{a_{i}}$ and $v=\prod_{i=1}^{n} x_{i}^{b_{i}}$ are monomials with $\sum_{i=1}^{n} a_{i}=\sum_{i=1}^{n} b_{i}$.) Let $<$ be a monomial order on $K[\mathbf{x}]$ and $\operatorname{in}_{<}\left(I_{A}\right)$ the initial ideal of $I_{A}$ with respect to $<$. We say that $\operatorname{in}_{<}\left(I_{A}\right)$ is squarefree if $\operatorname{in}_{<}\left(I_{A}\right)$ is generated by squarefree monomials. We refer the reader to [11, Chapters 1 and 5] for the information about Gröbner bases and toric ideals.

Let $\mathbb{Z}_{\geq 0}^{d}$ denote the set of integer column vectors $\left[a_{1}, \ldots, a_{d}\right]^{\top}$ with each $a_{i} \geq 0$. Given an integer vector $\mathbf{a}=\left[a_{1}, \ldots, a_{d}\right]^{\top} \in \mathbb{Z}^{d}$, let $\mathbf{a}^{(+)}=\left[a_{1}^{(+)}, \ldots, a_{d}^{(+)}\right]^{\top}, \mathbf{a}^{(-)}=$ $\left[a_{1}^{(-)}, \ldots, a_{d}^{(-)}\right]^{\top} \in \mathbb{Z}_{\geq 0}^{d}$ where $a_{i}^{(+)}=\max \left\{0, a_{i}\right\}$ and $a_{i}^{(-)}=\max \left\{0,-a_{i}\right\}$. Note that $\mathbf{a}=\mathbf{a}^{(+)}-\mathbf{a}^{(-)}$holds in general. Let $\mathbb{Z}_{\geq 0}^{d \times n}$ denote the set of $d \times n$ integer matrices $\left(a_{i j}\right)_{\substack{1 \leq i \leq d \\ 1 \leq j \leq n}}$ with each $a_{i j} \geq 0$. Furthermore if no columns of $A \in \mathbb{Z}_{\geq 0}^{d \times n}$ is the zero vector $\mathbf{0}=[0, \ldots, 0]^{\top} \in \mathbb{Z}^{d}$, then we introduce the $d \times(n+1)$ integer matrix $A^{\sharp}$ which is obtained by adding the column $\mathbf{0} \in \mathbb{Z}^{d}$ to $A$.

Now, given $A \in \mathbb{Z}_{\geq 0}^{d \times n}$ and $B \in \mathbb{Z}_{\geq 0}^{d \times m}$, we say that $A$ and $B$ are of harmony if the following condition is satisfied: Let a be a column of $A^{\sharp}$ and $\mathbf{b}$ that of $B^{\sharp}$. Let $\mathbf{c}=\mathbf{a}-\mathbf{b} \in \mathbb{Z}^{d}$. If $\mathbf{c}=\mathbf{c}^{(+)}-\mathbf{c}^{(-)}$, then $\mathbf{c}^{(+)}$is a column vector of $A^{\sharp}$ and $\mathbf{c}^{(-)}$is a column vector of $B^{\sharp}$.

Theorem 1.1. Let $A=\left[\mathbf{a}_{1}, \ldots, \mathbf{a}_{n}\right] \in \mathbb{Z}_{>0}^{d \times n}$ and $B=\left[\mathbf{b}_{1}, \ldots, \mathbf{b}_{m}\right] \in \mathbb{Z}_{>0}^{d \times m}$, where none of $\mathbf{a}_{i}$ 's and $\mathbf{b}_{j}$ 's is $\mathbf{0} \in \mathbb{Z}^{d}$, be of harmony. Let $K[z, \mathbf{x}]=K\left[z, x_{1}, \ldots, x_{n}\right]$ and $K[z, \mathbf{y}]=K\left[z, y_{1}, \ldots, y_{m}\right]$ be the polynomial rings over a field $K$. Suppose that $\operatorname{in}_{<_{A}}\left(I_{A^{\sharp}}\right) \subset K[z, \mathbf{x}]$ and $\operatorname{in}_{<_{B}}\left(I_{B^{\sharp}}\right) \subset K[z, \mathbf{y}]$ are squarefree with respect to reverse lexicographic orders $<_{A}$ on $K[z, \mathbf{x}]$ and $<_{B}$ on $K[z, \mathbf{y}]$ respectively satisfying the conditions that

- $x_{i}<_{A} x_{j}$ if $\pi\left(x_{i}\right)$ divides $\pi\left(x_{j}\right)$;

- $z<_{A} x_{k}$ for $1 \leq k \leq n$, where $z$ corresponds to the column $\mathbf{0} \in \mathbb{Z}^{d}$ of $A^{\sharp}$;

- $z<_{B} y_{k}$ for $1 \leq k \leq m$, where $z$ corresponds to the column $\mathbf{0} \in \mathbb{Z}^{d}$ of $B^{\sharp}$.

Let $[-B, A]$ denote the $d \times(n+m)$ integer matrix

$$
\left[-\mathbf{b}_{1}, \ldots,-\mathbf{b}_{m}, \mathbf{a}_{1}, \ldots, \mathbf{a}_{n}\right]
$$


Then the toric ideal $I_{[-B, A]}$ of $[-B, A]^{\sharp}$ possesses a squarefree initial ideal with respect to a reverse lexicographic order whose smallest variable corresponds to the column $\mathbf{0} \in \mathbb{Z}^{d}$ of $[-B, A]^{\sharp}$.

Proof. Let $K\left[[-B, A]^{\sharp}\right] \subset K\left[\mathbf{t}, \mathbf{t}^{-1}, s\right]=K\left[t_{1}, t_{1}^{-1}, \ldots, t_{d}, t_{d}^{-1}, s\right]$ be the toric ring of $[-B, A]^{\sharp}$ and $I_{[-B, A]^{\sharp}} \subset K[\mathbf{x}, \mathbf{y}, z]=K\left[x_{1}, \ldots, x_{n}, y_{1}, \ldots, y_{m}, z\right]$ the toric ideal of $[-B, A]^{\sharp}$. Recall that $I_{[-B, A]^{\sharp}}$ is the kernel of $\pi: K[\mathbf{x}, \mathbf{y}, z] \rightarrow K\left[[-B, A]^{\sharp}\right]$ with $\pi(z)=s, \pi\left(x_{i}\right)=\mathbf{t}^{\mathbf{a}_{i}} s$ for $i=1, \ldots, n$ and $\pi\left(y_{j}\right)=\mathbf{t}^{-\mathbf{b}_{j}} s$ for $j=1, \ldots, m$.

Suppose that the reverse lexicographic orders $<_{A}$ and $<_{B}$ are induced by the orderings $z<_{A} x_{n}<_{A} \cdots<_{A} x_{1}$ and $z<_{B} y_{m}<_{B} \cdots<_{B} y_{1}$. Let $<_{\text {rev }}$ be the reverse lexicographic order on $K[\mathbf{x}, \mathbf{y}, z]$ induced by the ordering

$$
z<x_{n}<\cdots<x_{1}<y_{m}<\cdots<y_{1} .
$$

In general, if $\mathbf{a}=\left[a_{1}, \ldots, a_{d}\right]^{\top} \in \mathbb{Z}_{\geq 0}^{d}$, then $\operatorname{supp}(\mathbf{a})$ is the set of those $1 \leq i \leq d$ with $a_{i} \neq 0$. Now, we introduce the following

$$
\mathcal{E}=\left\{(i, j): 1 \leq i \leq n, 1 \leq j \leq m, \operatorname{supp}\left(\mathbf{a}_{i}\right) \cap \operatorname{supp}\left(\mathbf{b}_{j}\right) \neq \emptyset\right\} .
$$

Let $\mathbf{c}=\mathbf{a}_{i}-\mathbf{b}_{j}$ with $(i, j) \in \mathcal{E}$. Then $\mathbf{c}^{(+)} \neq \mathbf{a}_{i}$ and $\mathbf{c}^{(-)} \neq \mathbf{b}_{j}$. The hypothesis that $A$ and $B$ are of harmony guarantees that $\mathbf{c}^{(+)}$is a column of $A^{\sharp}$ and $\mathbf{c}^{(-)}$is a column of $B^{\sharp}$. It follows that $f=x_{i} y_{j}-u(\neq 0)$ belongs to $I_{[-B, A]^{\sharp}}$, where

$$
u=\left\{\begin{array}{cl}
x_{k} y_{\ell} & \text { if } \mathbf{c}^{(+)}=\mathbf{a}_{k} \text { and } \mathbf{c}^{(-)}=\mathbf{b}_{\ell} \\
z y_{\ell} & \text { if } \mathbf{c}^{(+)}=\mathbf{0} \text { and } \mathbf{c}^{(-)}=\mathbf{b}_{\ell} \\
x_{k} z & \text { if } \mathbf{c}^{(+)}=\mathbf{a}_{k} \text { and } \mathbf{c}^{(-)}=\mathbf{0} \\
z^{2} & \text { if } \mathbf{c}^{(+)}=\mathbf{c}^{(-)}=\mathbf{0}
\end{array}\right.
$$

If $z$ divides $u$, then $\operatorname{in}_{<_{\text {rev }}}(f)=x_{i} y_{j}$, where $\operatorname{in}_{<_{\text {rev }}}(f)$ is the initial monomial of $f \in K[\mathbf{x}, \mathbf{y}, z]$. If $z$ cannot divide $u$, then, since $\pi\left(x_{k}\right)$ divides $\pi\left(x_{i}\right)$, one has $x_{k}<_{A} x_{i}$ and $\operatorname{in}_{<\text {rev }}(f)=x_{i} y_{j}$. Hence

$$
\left\{x_{i} y_{j}:(i, j) \in \mathcal{E}\right\} \subset \operatorname{in}_{<_{\text {rev }}}\left(I_{[-B, A]^{\sharp}}\right) .
$$

Now, let $\mathcal{M}_{A}$ (resp. $\mathcal{M}_{B}$ ) be the minimal set of squarefree monomial generators of $\operatorname{in}_{<_{A}}\left(I_{A^{\sharp}}\right)$ (resp. $\left.\operatorname{in}_{<_{B}}\left(I_{B^{\sharp}}\right)\right)$. Suppose that in ${ }_{<_{\text {rev }}}\left(I_{[-B, A]^{\sharp}}\right)$ cannot be generated by the set of squarefree monomials

$$
\mathcal{M}=\left\{x_{i} y_{j}:(i, j) \in \mathcal{E}\right\} \cup \mathcal{M}_{A} \cup \mathcal{M}_{B}\left(\subset \text { in }_{<_{\text {rev }}}\left(I_{[-B, A]^{\sharp}}\right)\right) .
$$

The following fact $([22,(0.1)$, p. 1914]) on Gröbner bases is known:

A finite set $\mathcal{G}$ of $I_{A}$ is a Gröbner basis with respect to $<$ if and only if $\pi(u) \neq \pi(v)$ for any monomials $u \notin\left(\operatorname{in}_{<}(g): g \in \mathcal{G}\right)$ and $v \notin\left(\operatorname{in}_{<}(g): g \in \mathcal{G}\right)$ with $u \neq v$.

Since $\mathcal{G}$ with $\mathcal{M}=\left\{\operatorname{in}_{<}(f): f \in \mathcal{G}\right\}$ is not a Gröbner basis, it follows that there exists a nonzero irreducible binomial $g=u-v$ belonging to $I_{[-B, A] \sharp}$ such that each 
of $u$ and $v$ can be divided by none of the monomials belonging to $\mathcal{M}$. Write

$$
u=\left(\prod_{p \in P} x_{p}^{i_{p}}\right)\left(\prod_{q \in Q} y_{q}^{j_{q}}\right), \quad v=z^{\alpha}\left(\prod_{r \in R} x_{r}^{k_{r}}\right)\left(\prod_{s \in S} y_{s}^{\ell_{s}}\right)
$$

where $P$ and $R$ are subsets of $\{1, \ldots, n\}$, where $Q$ and $S$ are subsets of $\{1, \ldots, m\}$, where $\alpha$ is a nonnegative integer, and where each of $i_{p}, j_{q}, k_{r}, \ell_{s}$ is a positive integer. Since $g=u-v$ is irreducible, one has $P \cap R=Q \cap S=\emptyset$. Furthermore, the fact that each of $x_{i} y_{j}$ with $(i, j) \in \mathcal{E}$ can divide neither $u$ nor $v$ guarantees that

$$
\left(\bigcup_{p \in P} \operatorname{supp}\left(\mathbf{a}_{p}\right)\right) \cap\left(\bigcup_{q \in Q} \operatorname{supp}\left(\mathbf{b}_{q}\right)\right)=\left(\bigcup_{r \in R} \operatorname{supp}\left(\mathbf{a}_{r}\right)\right) \cap\left(\bigcup_{s \in S} \operatorname{supp}\left(\mathbf{b}_{s}\right)\right)=\emptyset \text {. }
$$

Since $\pi(u)=\pi(v)$, it follows that

$$
\sum_{p \in P} i_{p} \mathbf{a}_{p}=\sum_{r \in R} k_{r} \mathbf{a}_{r}, \quad \sum_{q \in Q} j_{q} \mathbf{b}_{q}=\sum_{s \in S} \ell_{s} \mathbf{b}_{s} .
$$

Let $\gamma_{P}=\sum_{p \in P} i_{p}, \gamma_{Q}=\sum_{q \in Q} j_{q}, \gamma_{R}=\sum_{r \in R} k_{r}$, and $\gamma_{S}=\sum_{s \in S} \ell_{s}$. Then

$$
\gamma_{P}+\gamma_{Q}=\alpha+\gamma_{R}+\gamma_{S}
$$

Since $\alpha \geq 0$, it follows that either $\gamma_{P} \geq \gamma_{R}$ or $\gamma_{Q} \geq \gamma_{S}$. Let, say, $\gamma_{P}>\gamma_{R}$, then

$$
h=\prod_{p \in P} x_{p}^{i_{p}}-z^{\gamma_{P}-\gamma_{R}}\left(\prod_{r \in R} x_{r}^{k_{r}}\right) \neq 0
$$

belongs to $I_{[-B, A]^{\sharp}}$ and $\operatorname{in}_{<_{\text {rev }}}(h)=\prod_{p \in P} x_{p}^{i_{p}}$ divides $u$, a contradiction. Hence $\gamma_{P}=$ $\gamma_{R}$. Then the binomial

$$
h_{0}=\prod_{p \in P} x_{p}^{i_{p}}-\prod_{r \in R} x_{r}^{k_{r}}
$$

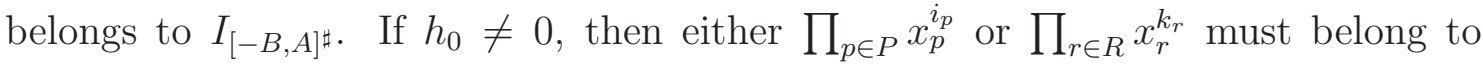
$\operatorname{in}_{<\text {rev }}\left(I_{[-B, A]^{\sharp}}\right)$. This contradicts the fact that each of $u$ and $v$ can be divided by none of the monomials belonging to $\mathcal{M}$. Hence $h_{0}=0$ and $P=R=\emptyset$. Similarly, $Q=S=\emptyset$. Hence $\alpha=0$ and $g=0$. This contradiction guarantees that $\mathcal{M}$ is the minimal set of squarefree monomial generators of $\operatorname{in}_{<_{\text {rev }}}\left(I_{[-B, A]^{\sharp}}\right)$, as desired.

Given an integral convex polytope $\mathcal{P} \subset \mathbb{R}^{d}$, we write $A_{\mathcal{P}}$ for the integer matrices whose column vectors are those $\mathbf{a} \in \mathbb{Z}^{d}$ belonging to $\mathcal{P}$. The toric ring $K\left[A_{\mathcal{P}}\right]$ is often called the toric ring of $\mathcal{P}$. A triangulation $\Delta$ of $\mathcal{P}$ with using the vertices belonging to $\mathcal{P} \cap \mathbb{Z}^{d}$ is unimodular if the normalized volume ([11, p. 253]) of each facet of $\Delta$ is equal to 1 and is flag if every minimal nonface of $\Delta$ is an edge. It follows from [26, Chapter 8] that if the toric ideal $I_{A_{\mathcal{P}}}$ of $A_{\mathcal{P}}$ possesses a squarefree initial ideal, then $\mathcal{P}$ possesses a unimodular triangulation. Furthermore if $I_{A_{\mathcal{P}}}$ possesses an initial ideal generated by quadratic squarefree monomials, then $\mathcal{P}$ possesses a unimodular triangulation which is flag. 
An integral convex polytope $\mathcal{P} \subset \mathbb{R}^{d}$ of dimension $d$ is called Fano if the origin of $\mathbb{R}^{d}$ is a unique integer point belonging to the interior of $\mathcal{P}$. We say that a Fano polytope $\mathcal{P}$ is Gorenstein if the dual polytope $\mathcal{P}^{\vee}$ of $\mathcal{P}$ is again integral ([2], [9]). A smooth Fano polytope is a simplicial Fano polytope $\mathcal{P} \subset \mathbb{R}^{d}$ for which the $d$ vertices of each facet of $\mathcal{P}$ is a $\mathbb{Z}$-basis of $\mathbb{Z}^{d}$.

Lemma 1.2. Let $\mathcal{P} \subset \mathbb{R}^{d}$ be an integral convex polytope of dimension $d$ for which $\mathbf{0} \in \mathbb{Z}^{d}$ belongs to $\mathcal{P}$. Suppose that there is a $d \times d$ minor $A^{\prime}$ of $A_{\mathcal{P}}$ with $\operatorname{det}\left(A^{\prime}\right)= \pm 1$ and that $I_{A_{\mathcal{P}}}$ possesses a squarefree initial ideal with respect to a reverse lexicographic order whose smallest variable corresponds to the column $\mathbf{0} \in \mathbb{Z}^{d}$ of $A_{\mathcal{P}}$. Then, for each facet $\mathcal{F}$ of $\mathcal{P}$ with $\mathbf{0} \notin \mathcal{F}$, one has $\mathbb{Z} \mathcal{F}=\mathbb{Z}^{d}$, where

$$
\mathbb{Z} \mathcal{F}=\sum_{\mathbf{a} \in \mathcal{F} \cap \mathbb{Z}^{d}} \mathbb{Z} \mathbf{a}
$$

and the equation of the supporting hyperplane $\mathcal{H} \subset \mathbb{R}^{d}$ with $\mathcal{F} \subset \mathcal{H}$ is of the form

$$
a_{1} z_{1}+\cdots+a_{d} z_{d}=1
$$

with each $a_{j} \in \mathbb{Z}$.

In particular if $\mathcal{P}$ is a Fano polytope, then $\mathcal{P}$ is Gorenstein. Furthermore if $\mathcal{P}$ is a simplicial Fano polytope, then $\mathcal{P}$ is a smooth Fano polytope.

Proof. Let $\Delta$ be the pulling triangulation ([11, p. 268]) coming from a squarefree initial ideal with respect to a reverse lexicographic order whose smallest variable corresponds to the column $\mathbf{0} \in \mathbb{Z}^{d}$ of $A_{\mathcal{P}}$. A crucial fact is that the origin of $\mathbb{R}^{d}$ belongs to each facet of $\Delta$. Let $F$ be a facet of $\Delta$ with the vertices $\mathbf{0}, \mathbf{b}_{1}, \ldots, \mathbf{b}_{d}$ for which $\left\{\mathbf{b}_{1}, \ldots, \mathbf{b}_{d}\right\} \subset \mathcal{F}$. The existence of a $d \times d$ minor $A^{\prime}$ of $A_{\mathcal{P}}$ with $\operatorname{det}\left(A^{\prime}\right)= \pm 1$ guarantees that the normalized volume of $F$ coincides with $|\operatorname{det}(B)|$, where $B=$ $\left[\mathbf{b}_{1}, \ldots, \mathbf{b}_{d}\right]$. Since $\Delta$ is unimodular, one has $\operatorname{det}(B)= \pm 1$. Hence $\left\{\mathbf{b}_{1}, \ldots, \mathbf{b}_{d}\right\}$ is a $\mathbb{Z}$-basis of $\mathbb{Z}^{d}$ and $\mathbb{Z} \mathcal{F}=\mathbb{Z}^{d}$ follows. Moreover the hyperplane $\mathcal{H} \subset \mathbb{R}^{d}$ with each $\mathbf{b}_{j} \in \mathcal{H}$ is of the form $a_{1} z_{1}+\cdots+a_{d} z_{d}=1$ with each $a_{j} \in \mathbb{Z}$, as desired.

Corollary 1.3. Work with the same situation as in Theorem 1.1. Let $\mathcal{P} \subset \mathbb{R}^{d}$ be the integral convex polytope which is the convex hull of $\left\{-\mathbf{b}_{1}, \ldots,-\mathbf{b}_{m}, \mathbf{a}_{1}, \ldots, \mathbf{a}_{n}\right\}$. Suppose that $\mathbf{0} \in \mathbb{Z}^{d}$ belongs to the interior of $\mathcal{P}$ and that there is a $d \times d$ minor $A^{\prime}$ of $A_{\mathcal{P}}$ with $\operatorname{det}\left(A^{\prime}\right)= \pm 1$. Then $\mathcal{P}$ is a Gorenstein Fano polytope. Furthermore if $\mathcal{P}$ is a simplicial polytope, then $\mathcal{P}$ is a smooth Fano polytope.

Example 1.4. Let $A_{1}$ and $A_{2}$ be the following matrices:

$$
A_{1}=\left[\begin{array}{ll}
1 & 0 \\
0 & 1
\end{array}\right], \quad A_{2}=\left[\begin{array}{lll}
1 & 0 & 1 \\
0 & 1 & 1
\end{array}\right] .
$$

Then, $A_{i}$ and $A_{j}$ are of harmony and satisfy the condition in Theorem 1.1 for any $1 \leq i \leq j \leq 2$. By Corollary 1.3, we have three Gorenstein Fano polygons. It is known that there are exactly 16 Gorenstein Fano polygons ([5, p.382]). 


\section{Convex polytopes ARISING FROM Simplicial COMPleXes}

Let $[d]=\{1, \ldots, d\}$ and $\mathbf{e}_{1}, \ldots, \mathbf{e}_{d}$ the standard coordinate unit vectors of $\mathbb{R}^{d}$. Given a subset $W \subset[d]$, one has $\rho(W)=\sum_{j \in W} \mathbf{e}_{j} \in \mathbb{R}^{d}$. In particular $\rho(\emptyset)$ is the origin of $\mathbb{R}^{d}$. Let $\Delta$ be a simplicial complex on the vertex set $[d]$. Thus $\Delta$ is a collection of subsets of $[d]$ with $\{i\} \in \Delta$ for each $i \in[d]$ such that if $F \in \Delta$ and $F^{\prime} \subset F$, then $F^{\prime} \in \Delta$. In particular $\emptyset \in \Delta$. The incidence matrix $A_{\Delta}$ of $\Delta$ is the matrix whose columns are those $\rho(F)$ with $F \in \Delta$. We write $\mathcal{P}_{\Delta} \subset \mathbb{R}^{d}$ for the $(0,1)$-polytope which is the convex hull of $\{\rho(F): F \in \Delta\}$ in $\mathbb{R}^{d}$. One has $\operatorname{dim} \mathcal{P}_{\Delta}=d$. It follows from the definition of simplicial complexes that

Lemma 2.1. Let $\Delta$ and $\Delta^{\prime}$ be simplicial complexes on $[d]$. Then $A_{\Delta}$ and $A_{\Delta^{\prime}}$ are of harmony.

Following Lemma 2.1 together with Theorem 1.1, it is reasonable to study the problem when the toric ideal $I_{A_{\Delta}}$ of a simplicial complex $\Delta$ possesses a squarefree initial ideal with respect to a reverse lexicographic order whose smallest variable corresponds to the column $\mathbf{0} \in \mathbb{Z}^{d}$ of $A_{\Delta}$.

Let $\Delta$ be a simplicial complex on $[d]$. Since $\{i\} \in \Delta$ for each $i \in[d]$, the $d \times d$ identity matrix is a $d \times d$ minor of $A_{\Delta}$. It then follows from Lemma 1.2 that

Corollary 2.2. Let $\Delta$ be a simplicial complex on $[d]$. Suppose that $I_{A_{\Delta}}$ possesses a squarefree initial ideal with respect to a reverse lexicographic order whose smallest variable corresponds to the column $\mathbf{0} \in \mathbb{Z}^{d}$ of $A_{\Delta}$. Then, for each facet $\mathcal{F}$ of $\mathcal{P}_{\Delta}$ with $\mathbf{0} \notin \mathcal{F}$, one has $\mathbb{Z} \mathcal{F}=\mathbb{Z}^{d}$ and the equation of the supporting hyperplane $\mathcal{H} \subset \mathbb{R}^{d}$ with $\mathcal{F} \subset \mathcal{H}$ is of the form $a_{1} z_{1}+\cdots+a_{d} z_{d}=1$ with each $a_{j} \in \mathbb{Z}$.

Let $G$ be a finite simple graph on $[d]$ and $E(G)$ the set of edges of $G$. (Recall that a finite graph is simple if $G$ possesses no loop and no multiple edge.) A subset $W \subset[d]$ is called stable if, for all $i$ and $j$ belonging to $W$ with $i \neq j$, one has $\{i, j\} \notin E(G)$. Let $S(G)$ denote the set of stable sets of $G$. One has $\emptyset \in S(G)$ and $\{i\} \in S(G)$ for each $i \in[d]$. Clearly $S(G)$ is a simplicial complex on $[d]$. The stable set polytope $\mathcal{Q}_{G} \subset \mathbb{R}^{d}$ of $G$ is the $(0,1)$-polytope $\mathcal{P}_{S(G)} \subset \mathbb{R}^{d}$ arising from the simplicial complex $S(G)$. A finite simple graph is said to be perfect ([3]) if, for any induced subgraph $H$ of $G$ including $G$ itself, the chromatic number of $H$ is equal to the maximal cardinality of cliques of $H$. (A chromatic number of $G$ is the smallest integer $t$ for which there exist stable set $W_{1}, \ldots, W_{t}$ of $G$ with $[d]=W_{1} \cup \cdots \cup W_{t}$ and a clique of $G$ is a subset $W \subset[d]$ which is a stable set of the complementary graph $\bar{G}$ of $G$.) A complementary graph of a perfect graph is perfect ([3]).

Recall that an integer matrix $A$ is compressed ([21], [27]) if the initial ideal of the toric ideal $I_{A}$ is squarefree with respect to any reverse lexicographic order.

Example 2.3. Let $G$ be a perfect graph on $[d]$. Then $A_{\Delta}$, where $\Delta=S(G)$, is compressed ([21, Example $1.3(\mathrm{c})])$. Let $G$ and $G^{\prime}$ be perfect graphs on $[d]$ and 
$\mathcal{Q} \subset \mathbb{R}^{d}$ be the Fano polytope which is the convex hull of $\mathcal{Q}_{G} \cup\left(-\mathcal{Q}_{G^{\prime}}\right)$. It then follows from Corollary 1.3 together with Lemma 2.1 that $\mathcal{Q}$ is Gorenstein.

Lemma 2.4. Let $\Delta$ be one of the following simplicial complexes:

(i) the simplicial complex on $[e]$ with the facets $[e] \backslash\{i\}, 1 \leq i \leq e$, where $e \geq 3$;

(ii) $S(G)$, where $G$ is an odd hole of length $2 \ell+1$, where $\ell \geq 2$;

(iii) $S(G)$, where $G$ is an odd antihole of length $2 \ell+1$, where $\ell \geq 2$;

Let $<$ be any reverse lexicographic order whose smallest variable corresponds to the column 0 of $A_{\Delta}$. Then the initial ideal in in $_{<}\left(I_{A_{\Delta}}\right)$ cannot be squarefree. (Recall that an odd hole is an induced odd cycle of length $\geq 5$ and an odd antihole is the complementary graph of an odd hole.)

Proof. By virtue of Corollary 2.2, we find a supporting hyperplane $\mathcal{H}$ of $\mathcal{P}_{\Delta}$ with $\mathbf{0} \notin \mathcal{H}$ for which $\mathcal{H} \cap \mathcal{P}_{\Delta}$ is a facet of $\mathcal{P}_{\Delta}$ such that the equation of $\mathcal{H}$ cannot be of the form $a_{1} z_{1}+\cdots+a_{d} z_{d}=1$ with each $a_{j} \in \mathbb{Z}$. In each of (i), (ii) and (iii), the equation of a desired hyperplane $\mathcal{H}$ is as follows:

(i) $\sum_{i=1}^{e} z_{i}=e-1$;

(ii) $\sum_{i=1}^{2 \ell+1} z_{i}=\ell$;

(iii) $\sum_{i=1}^{2 \ell+1} z_{i}=2$.

In (i), it is easy to see that $\mathcal{H} \cap \mathcal{P}_{\Delta}$ is a facet of $\mathcal{P}_{\Delta}$. In each of (ii) and (iii), it is known $([25]$, [28] $)$ that $\mathcal{H} \cap \mathcal{P}_{\Delta}$ is a facet of $\mathcal{P}_{\Delta}$.

Let $B=\left[\mathbf{b}_{1}, \ldots, \mathbf{b}_{m}\right] \in \mathbb{Z}^{d \times m}$ be a submatrix of $A=\left[\mathbf{a}_{1}, \ldots, \mathbf{a}_{n}\right] \in \mathbb{Z}^{d \times n}$. Then, $K[B]$ is called a combinatorial pure subring of $K[A]$ if the convex hull of $\left\{\mathbf{b}_{1}, \ldots, \mathbf{b}_{m}\right\}$ is a face of the convex hull of $\left\{\mathbf{a}_{1}, \ldots, \mathbf{a}_{n}\right\}$. For any combinatorial pure subring $K[B]$ of $K[A]$, it is known that, if the initial ideal of $I_{A}$ is squarefree, then so is the corresponding initial ideal of $I_{B}$. See [20, 19] for details.

Lemma 2.5. Let $\Delta$ be a simplicial complex on $[d]$ and $\Delta^{\prime}$ an induced subcomplex of $\Delta$ which is one of (i), (ii) and (iii) of Lemma 2.4. Let $<$ be any reverse lexicographic order whose smallest variable corresponds to the column $\mathbf{0} \in \mathbb{Z}^{d}$ of $A_{\Delta}$. Then the initial ideal $\operatorname{in}_{<}\left(I_{A_{\Delta}}\right)$ cannot be squarefree.

Proof. Let $\Delta^{\prime}$ be the induced subcomplex of $\Delta$ on $V$, where $V \subset[d]$, and $<^{\prime}$ the reverse lexicographic order induced by $<$. Lemma 2.4 says that $\operatorname{in}_{<^{\prime}}\left(I_{A_{\Delta^{\prime}}}\right)$ cannot be squarefree. Since $\Delta^{\prime}$ is an induced subcomplex of $\Delta$, it follows that $\mathcal{P}_{\Delta^{\prime}}$ is a face of $\mathcal{P}_{\Delta}$. Thus $K\left[A_{\Delta^{\prime}}\right]$ is a combinatorial pure subring of $K\left[A_{\Delta}\right]$ and hence $\operatorname{in}_{<}\left(I_{A_{\Delta}}\right)$ cannot be squarefree, as required.

We are now in the position to state a combinatorial characterization of simplicial complexes $\Delta$ on $[d]$ for which the toric ideal $I_{A_{\Delta}}$ possesses a squarefree initial ideal with respect to a reverse lexicographic order whose smallest variable corresponds to the column $\mathbf{0} \in \mathbb{Z}^{d}$ of $A_{\Delta}$. 
Theorem 2.6. Let $\Delta$ be a simplicial complex on $[d]$. Then the following conditions are equivalent:

(i) There exists a perfect graph $G$ on $[d]$ with $\Delta=S(G)$;

(ii) $A_{\Delta}$ is compressed;

(iii) $I_{A_{\Delta}}$ possesses a squarefree initial ideal with respect to a reverse lexicographic order whose smallest variable corresponds to the column $\mathbf{0} \in \mathbb{Z}^{d}$ of $A_{\Delta}$.

Proof. In [21, Example 1.3 (c)], (i) $\Rightarrow$ (ii) is proved. (See also [6, §4].) Moreover, (ii) $\Rightarrow$ (iii) is trivial. Now, in order to show (iii) $\Rightarrow$ (i), we fix a reverse lexicographic order $<$ whose smallest variable corresponds $\mathbf{0} \in \mathbb{Z}^{d}$ of $A_{\Delta}$.

(First Step) Suppose that there is no finite simple graph $G$ on $[d]$ with $\Delta=S(G)$. Given a simplicial complex $\Delta$ on $[d]$, there is a finite simple graph $G$ on $[d]$ with $\Delta=S(G)$ if and only if $\Delta$ is flag, i.e, every minimal nonface of $\Delta$ is an edge of $\Delta$. (See, e.g., [8, Lemma 9.1.3]. Note that $S(G)$ is the clique complex of the complement graph of $G$.) Let $\Delta$ be a simplicial complex which is not flag and $V \subset[d]$, where $|V| \geq 3$, a minimal nonface of $\Delta$. One has $V \backslash\{i\} \in \Delta$ for all $i \in V$. Thus the induced subcomplex $\Delta^{\prime}$ of $\Delta$ on $V$ coincides with the simplicial complex (i) of Lemma 2.4.

(Second Step) Let $G$ be a nonperfect graph on $[d]$ with $A_{\Delta}=S(G)$. The strong perfect graph theorem [3] guarantees that $G$ possesses either an odd hole or an odd antihole. Thus $\Delta$ contains an induced subcomplex $\Delta^{\prime}$ which coincides with either (ii) or (iii) of Lemma 2.4.

As a result, Lemma 2.5 says that $I_{A_{\Delta}}$ possesses no squarefree initial ideal with respect to a reverse lexicographic order whose smallest variable corresponds to the column $\mathbf{0} \in \mathbb{Z}^{d}$ of $A_{\Delta}$. This completes the proof of (iii) $\Rightarrow$ (i).

Example 2.7. Let $A \in \mathbb{Z}^{d \times n}$ for which each entry of $A$ belongs to $\{0,1\}$ and $I_{A^{\sharp}}$ the toric ideal of $A^{\sharp}$. In general, even if $I_{A^{\sharp}}$ possesses a squarefree initial ideal with respect to a reverse lexicographic order whose smallest variable corresponds to the column $\mathbf{0} \in \mathbb{Z}^{d}$ of $A^{\sharp}$, the matrix $A^{\sharp}$ may not be compressed. For example, if

$$
A=\left[\begin{array}{lllllll}
1 & 0 & 1 & 1 & 0 & 0 & 0 \\
1 & 1 & 0 & 0 & 0 & 0 & 0 \\
0 & 1 & 1 & 0 & 0 & 0 & 0 \\
0 & 0 & 0 & 1 & 1 & 0 & 1 \\
0 & 0 & 0 & 0 & 1 & 1 & 0 \\
0 & 0 & 0 & 0 & 0 & 1 & 1
\end{array}\right],
$$

then $I_{A^{\sharp}}$ is generated by $x_{1} x_{3} x_{5} x_{7}-x_{2} x_{4}^{2} x_{6}$. Thus the initial ideals of $I_{A^{\sharp}}$ with respect to the reverse lexicographic order induced by the ordering

$$
z<x_{2}<x_{1}<x_{3}<x_{4}<x_{5}<x_{6}<x_{7}
$$


is generated by $x_{1} x_{3} x_{5} x_{7}$, while the initial ideals of $I_{A} \sharp$ with respect to the reverse lexicographic order induced by the ordering

$$
z<x_{1}<x_{2}<x_{3}<x_{4}<x_{5}<x_{6}<x_{7}
$$

is generated by $x_{2} x_{4}^{2} x_{6}$. Even though $A^{\sharp}$ satisfies the condition (iii) of Theorem 2.6. the integer matrix $A^{\sharp}$ cannot be compressed.

Apart from Theorem 2.6, we can ask the problem when the convex polytope $\mathcal{P} \subset \mathbb{R}^{d}$ which is the convex hull of $\mathcal{P}_{\Delta} \cup\left(-\mathcal{P}_{\Delta^{\prime}}\right)$, where $\Delta$ and $\Delta^{\prime}$ are simplicial complexes on $[d]$, is a Gorenstein Fano polytope.

Theorem 2.8. Let $\Delta$ and $\Delta^{\prime}$ be simplicial complexes on $[d]$ and $\mathcal{P} \subset \mathbb{R}^{d}$ the convex polytope which is the convex hull of $\mathcal{P}_{\Delta} \cup\left(-\mathcal{P}_{\Delta^{\prime}}\right)$. Then $\mathcal{P}$ is a Gorenstein Fano polytope if and only if there exist perfect graphs $G$ and $G^{\prime}$ on $[d]$ with $\Delta=S(G)$ and $\Delta^{\prime}=S\left(G^{\prime}\right)$

Proof. The "If" part follows from Example 2.3. To see why the "Only If" part is true, suppose that either $\Delta$ is not flag or there is a nonperfect graph $G$ with $\Delta=S(G)$. Since $\mathcal{P} \subset \mathbb{R}^{d}$ is a Gorenstein Fano polytope, the equation of the supporting hyperplane $\mathcal{H} \subset \mathbb{R}^{d}$ for which $\mathcal{H} \cap \mathcal{P}$ is a facet of $\mathcal{P}$ is of the form $a_{1} z_{1}+\cdots+a_{d} z_{d}=1$ with each $a_{j} \in \mathbb{Z}$.

Let $\Delta$ be not flag and $V \subset[d]$ with $|V| \geq 3$ for which $V \backslash\{i\} \in \Delta$ for all $i \in V$ and $V \notin \Delta$. Let, say, $V=[e]$ with $e \geq 3$. Then the hyperplane $\mathcal{H}^{\prime} \subset \mathbb{R}^{d}$ defined by the equation $z_{1}+\cdots+z_{e}=e-1$ is a supporting hyperplane of $\mathcal{P}$. Let $\mathcal{F}$ be a facet of $\mathcal{P}$ with $\mathcal{H}^{\prime} \cap \mathcal{P} \subset \mathcal{F}$ and $a_{1} z_{1}+\cdots+a_{d} z_{d}=1$ with each $a_{j} \in \mathbb{Z}$ the equation of the supporting hyperplane $\mathcal{H} \subset \mathbb{R}^{d}$ with $\mathcal{F} \subset \mathcal{H}$. Since $\rho(V \backslash\{i\}) \in \mathcal{H}$ for all $i \in V$, one has $\sum_{j \in[e] \backslash\{i\}} a_{j}=1$. Thus $(e-1)\left(a_{1}+\cdots+a_{e}\right)=e$. Hence $a_{1}+\cdots+a_{e} \notin \mathbb{Z}$, a contradiction.

Let $\Delta=S(G)$, where $G$ possesses an odd hole $C$ of length $2 \ell+1$ with the vertices, say, $1, \ldots, 2 \ell+1$, where $\ell \geq 2$. Then the hyperplane $\mathcal{H}^{\prime} \subset \mathbb{R}^{d}$ defined by the equation $z_{1}+\cdots+z_{2 \ell+1}=\ell$ is a supporting hyperplane of $\mathcal{P}$. Let $\mathcal{F}$ be a facet of $\mathcal{P}$ with $\mathcal{H}^{\prime} \cap \mathcal{P} \subset \mathcal{F}$ and $a_{1} z_{1}+\cdots+a_{d} z_{d}=1$ with each $a_{j} \in \mathbb{Z}$ the equation of the supporting hyperplane $\mathcal{H} \subset \mathbb{R}^{d}$ with $\mathcal{F} \subset \mathcal{H}$. The maximal stable sets of $C$ is

$$
\{1,3, \ldots, 2 \ell-1\},\{2,4, \ldots, 2 \ell\}, \ldots,\{2 \ell+1,2,4, \ldots, 2 \ell-2\}
$$

and each $i \in[2 \ell-2]$ appears $\ell$ times in the above list. Since, for each maximal stable set $U$ of $C$, one has $\sum_{i \in U} a_{i}=1$, it follows that $\ell\left(a_{1}+\cdots+a_{2 \ell+1}\right)=2 \ell+1$. Hence $a_{1}+\cdots+a_{e} \notin \mathbb{Z}$, a contradiction.

Let $\Delta=S(G)$, where $G$ possesses an odd antihole $C$ with the vertices, say, $1, \ldots, 2 \ell+1$, where $\ell \geq 2$. Then the hyperplane $\mathcal{H}^{\prime} \subset \mathbb{R}^{d}$ defined by the equation $z_{1}+\cdots+z_{2 \ell+1}=2$ is a supporting hyperplane of $\mathcal{P}$. Let $\mathcal{F}$ be a facet of $\mathcal{P}$ with $\mathcal{H}^{\prime} \cap \mathcal{P} \subset \mathcal{F}$ and $a_{1} z_{1}+\cdots+a_{d} z_{d}=1$ with each $a_{j} \in \mathbb{Z}$ the equation of the supporting 
hyperplane $\mathcal{H} \subset \mathbb{R}^{d}$ with $\mathcal{F} \subset \mathcal{H}$. The maximal stable sets of $C$ is

$$
\{1,2\},\{2,3\}, \ldots,\{2 \ell+1,1\}
$$

and each $i \in[2 \ell-2]$ appears twice in the above list. Since, for each maximal stable set $U$ of $C$, one has $\sum_{i \in U} a_{i}=1$, it follows that $2\left(a_{1}+\cdots+a_{2 \ell+1}\right)=2 \ell+1$. Hence $a_{1}+\cdots+a_{e} \notin \mathbb{Z}$, a contradiction.

Acknowledgment. The authors are grateful to an anonymous referee for useful suggestions and helpful comments.

\section{REFERENCES}

[1] C. A. Athanasiadis, Ehrhart polynomials, simplicial polytopes, magic squares and a conjecture of Stanley, J. Reine Angew. Math. 583 (2005), 163-174.

[2] V. Batyrev, Dual polyhedra and mirror symmetry for Calabi-Yau hypersurfaces in toric varieties, J. Algebraic Geom. 3 (1994), 493-535.

[3] M. Chudnovsky, N. Robertson, P. Seymour and R. Thomas, The strong perfect graph theorem, Ann. of Math. 164 (2006), 51-229.

[4] H. Conrads, Weighted projective spaces and reflexive simplices, Manuscripta Math. 107 (2002), 215-227.

[5] D. Cox, J. Little and H. Schenck, "Toric Varieties," Amer. Math. Soc., Providence, RI, 2011.

[6] J. Gouveia, P. A. Parrilo and R. R. Thomas, Theta bodies for polynomial ideals, SIAM Journal on Optimization 20 (2010), 2097-2118.

[7] G. Hegedüs and A. M. Kasprzyk, The boundary volume of a lattice polytope, Bull. Aust. Math. Soc. 85 (2012), 84-104.

[8] J. Herzog and T. Hibi, "Monomial ideals," Grad. Texts in Math. 260, Springer, London, 2010.

[9] T. Hibi, Dual polytopes of rational convex polytopes, Combinatorica 12 (1992), 237-240.

[10] T. Hibi, Toric varieties arising from canonical triangulations of poset polytopes are projective, in "Words, Languages and Combinatorics II" (M. Ito and H. Jurgensen, Eds.), World Scientific, Singapore, 1994, pp. 192 - 199.

[11] T. Hibi, Ed., "Gröbner Bases: Statistics and Software Systems," Springer, 2013.

[12] T. Hibi, K. Matsuda, H. Ohsugi and K. Shibata, Centrally symmetric configurations of order polytopes, J. Algebra 443 (2015), 469-478.

[13] A. Higashitani, Smooth Fano polytopes arising from finite directed graphs, Kyoto J. Math. 55 (2015), 579-592.

[14] A. M. Kasprzyk, Classifying terminal weighted projective space, preprint, 2013. arXiv:1304.3029.

[15] A. M. Kasprzyk and B. Nill, Fano polytopes, in "Strings, Gauge Fields, and the Geometry Behind - the Legacy of Maximilian Kreuzer" (A. Rebhan, L. Katzarkov, J. Knapp, R. Rashkov, E. Scheidegger, Eds.), World Scientific, 2012, pp. 349-364.

[16] M. Kreuzer and H. Skarke, Classification of reflexive polyhedra in three dimensions, Adv. Theor. Math. Phys. 2 (1998), no. 4, 853-871.

[17] M. Kreuzer and H. Skarke, Complete classification of reflexive polyhedra in four dimensions, Adv. Theor. Math. Phys. 4 (2000), no. 6, 1209-1230.

[18] B. Nill, Volume and lattice points of reflexive simplices, Discrete Comput. Geom. 37 (2007), 301-320. 
[19] H. Ohsugi, A geometric definition of combinatorial pure subrings and Gröbner bases of toric ideals of positive roots, Comment. Math. Univ. St. Pauli 56 (2007), 27-44.

[20] H. Ohsugi, J. Herzog and T. Hibi, Combinatorial pure subrings, Osaka J. Math 37 (2000), $745-757$.

[21] H. Ohsugi and T. Hibi, Convex polytopes all of whose reverse lexicographic initial ideals are squarefree, Proc. Amer. Math. Soc. 129 (2001), 2541-2546.

[22] H. Ohsugi and T. Hibi, Quadratic initial ideals of root systems, Proc. Amer. Math. Soc. 130 (2002), 1913-1922.

[23] H. Ohsugi and T. Hibi, Centrally symmetric configurations of integer matrices, Nagoya Math. J. 216 (2014), 153-170.

[24] R. P. Stanley, "Combinatorics and Commutative Algebra," Second Ed., Birkhäuser, 1996.

[25] M. W. Padberg, Perfect zero-one matrices, Math. Programming 6 (1974), 180-196.

[26] B. Sturmfels, "Gröbner bases and convex polytopes," Amer. Math. Soc., Providence, RI, 1996.

[27] S. Sullivant, Compressed polytopes and statistical disclosure limitation, Tohoku Math. J. 58 (2006), $433-445$.

[28] A. Wagler, Critical edges in perfect line graphs and some polyhedral consequences, Disc. Appl. Math. 95 (1999), 455-466.

Hidefumi Ohsugi, Department of Mathematical Sciences, School of Science and Technology, Kwansei Gakuin University, Sanda, Hyogo, 669-1337, Japan

E-mail address: ohsugi@kwansei.ac.jp

Takayuki Hibi, Department of Pure and Applied Mathematics, Graduate School of Information Science and Technology, Osaka University, Toyonaka, Osaka 5600043, JAPAN

E-mail address: hibi@math.sci.osaka-u.ac.jp 